\title{
Extraluminal hypopharyngeal foreign body
}

\author{
Raman Vadhera - S. P. Gulati · Ajay Garg · Rohit Goyal · Anju Ghai
}

\begin{abstract}
Ingestion of a foreign body is a problem seen in nearly all otolaryngologic practices. Ingested foreign bodies which migrate extraluminally are rare occurrences. If untreated, they may result in life threatening complications. We are reporting a case of sharp metallic pin which migrated extraluminally in the hypopharynx and became lodged in the soft tissue. It was successfully removed by direct laryngoscopy.
\end{abstract}

Keywords Extra luminal - Foreign Body • Hypopharynx

R. Vadhera - S. P. Gulati - A. Garg • R. Goyal • A. Ghai

Department of Otorhinolaryngology \& Anaesthesiology, PT. BDS. PGIMS, Rohtak,

Haryana, India

A. Garg $(\bowtie)$

E mail: dr_ajay_garg@yahoo.co.in

\section{Introduction}

A swallowed foreign body can become embedded in the tonsil, base of the tongue, piriform fossae, or any area of the upper esophagus. These are easily removed by laryngoscopy or esophagoscopy. An even smaller fraction of cases occur in which the foreign body penetrates the wall and "migrates" through the deeper layers. In some instances, the foreign body can migrate completely through the wall and become lodged in the soft tissues of the neck $[1,2]$. There have been cases reported in which the foreign body actually exits through a puncture wound in the skin of the neck [3]. The term "migrating foreign bodies" has been coined for such cases. Prompt diagnosis is essential to the management of a perforating or migrating foreign body. The specific nature of symptoms, of course, is very helpful in localizing the site of the foreign body [4]. A finding of a laceration, edema, or ulceration on direct laryngoscopy and esophagoscopy should raise the level of suspicion for a perforating foreign body. Some authors advocate laryngoscopic microscopy to visualize and remove a foreign body [5]. X-ray is an important tool for localizing a perforating foreign body in the neck, but it can be misleading in cases where the cartilages of the upper airway are calcified. Computed tomography (CT) is considered to be the study of choice in such cases.

\section{Case report}

A 21-year-old woman initially presented to the otorhinolaryngology Department complaining that she felt a sudden sharp pain in her throat while taking dinner followed by persistent foreign body sensation on left side. On examination his general condition was satisfactory. Vital parameters were well maintained. Throat examination was normal. Indirect laryngoscopy did not reveal any pooling of saliva, foreign body, oedema or any congestion. Laryngeal inlet and larynx were normal. Neck movements were painless. 
There was no sign of trauma, swelling or tenderness and laryngeal crepitus was present. Systemic examination was essentially normal. Patient was investigated. Lateral softtissue radiograph of neck showed a thin foreign body $5 \mathrm{~cm}$ in length lying in an oblique plane at level of C5 vertebra (Fig. 1). On anteroposterior film, the foreign body was displaced from the midline, on the left side suggesting an extra luminal position (Fig. 2). Direct laryngoscopy under general anesthesia was performed. A small ulcer was seen on the lateral wall of piriform fossa, on end of foreign body was visible through that ulcer. It was grasped and removed with the help of forceps. The patient had apparently swallowed the wire somehow while having dinner. Her symptoms of foreign body sensation disappeared in immediate post operative period and she became totally asymptomatic.

\section{Discussion}

Oesophageal and hypopharyngeal foreign bodies are usually found intraluminally. There have been very few interesting reports of pharyngeal and oesophageal foreign bodies at

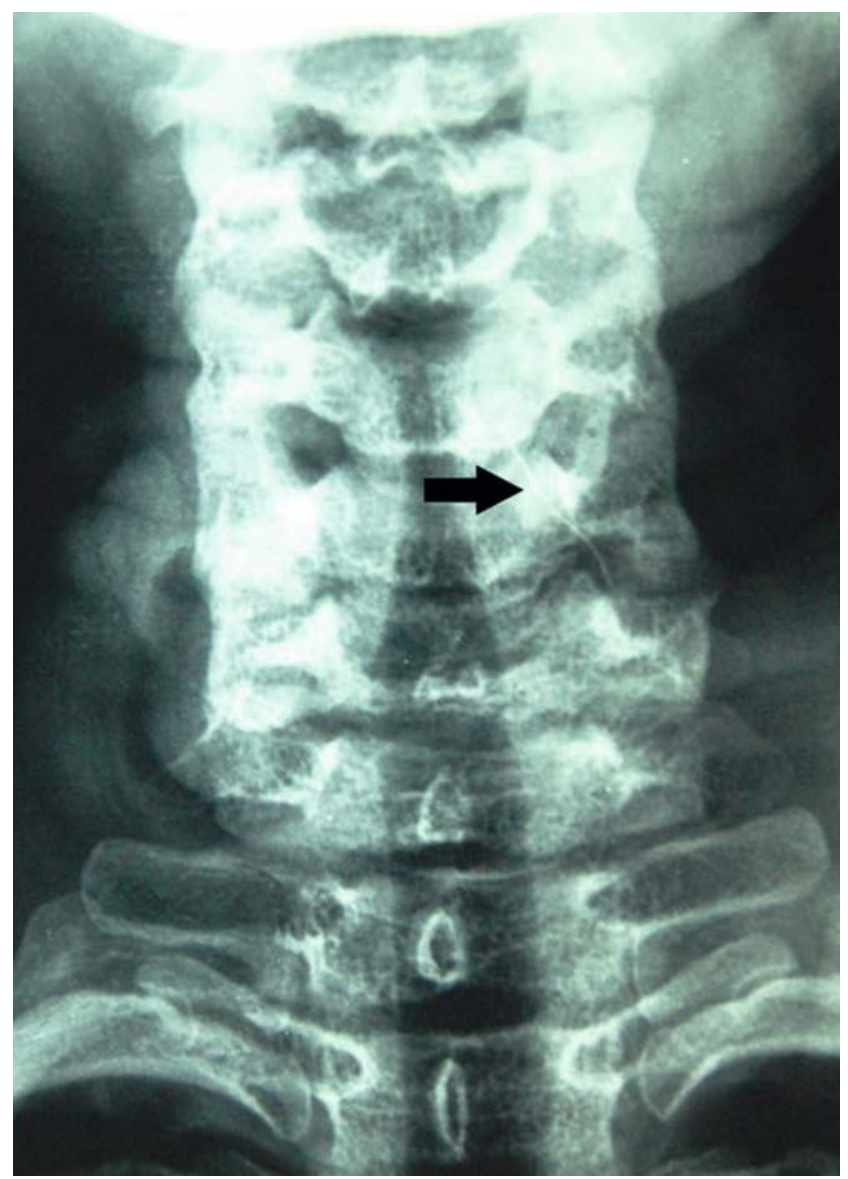

Fig. 1 A photo shows radiograph of the soft tissues of neck, lateral view, showing a radio opaque wire-like foreign body anterior to the body of the fifth cervical vertebra. sites other than the lumen. The number of ingested foreign bodies that perforate the aero digestive tract is small, and an even smaller number of foreign bodies migrate extraluminally. The sharper the foreign body is, the higher the risk of penetration. The risk of penetration is also influenced by the foreign body's orientation; horizontally oriented foreign bodies are more likely to penetrate. When perforation occurs, it is facilitated by the strong contraction of the hypopharyngeal and cricoesophageal muscles as they propel a food bolus into the oesophagus; this explains why higher rates of penetration occur in the hypopharynx and cervical oesophagus. The duration of impaction may not have a strong influence on the risk of penetration. ${ }^{6}$ In Remsen et al's series, in which 321 cases of penetrating foreign bodies were reviewed from the literature, only 43 were found extraluminally [3].

In patients with penetrating foreign bodies may be asymptomatic initially, but most eventually present with a foreign-body sensation, a sharp pricking sensation on swallowing, odynophagia, and/or hemoptysis. A migrated foreign body may remain quiescent or cause life-threatening suppurative or vascular complications. Perforating and

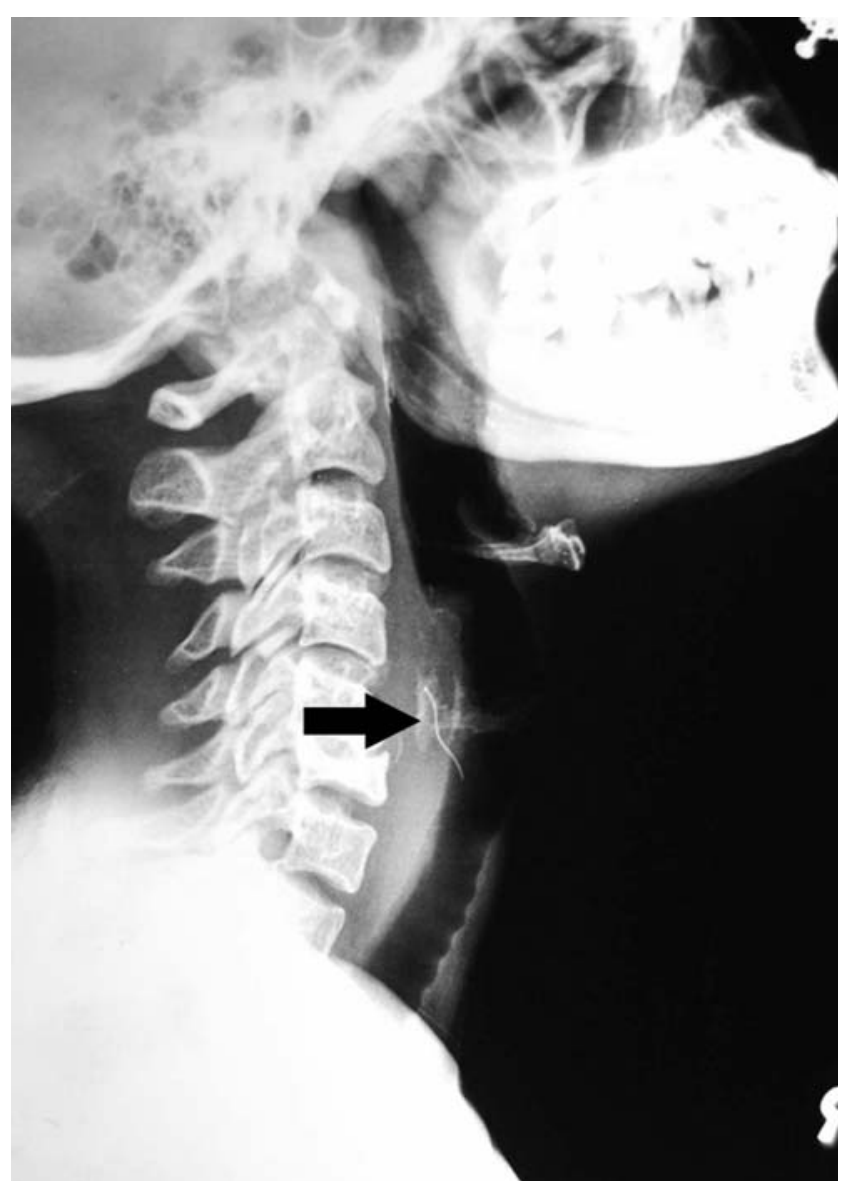

Fig. 2 A photo shows radiograph of the soft tissues of neck, anterior-posterior view, showing a radio opaque wire-like foreign body lying extraluminally in oblique direction 
migrating foreign bodies introduce bacteria into the soft tissue of the neck and cause suppurative complications such as para- or retropharyngeal abscess. Infection can spread into the mediastinum and lead to life-threatening mediastinitis. A foreign body might also penetrate adjacent visceral structures such as the thyroid gland. Finally, these objects can also penetrate the major blood vessels in the neck and precipitate vascular complications such as aortoesophageal and innominate-esophageal fistulae and carotid rupture [7].

One of the most common tools used to identify foreign bodies is the lateral neck x-ray. Pathology can be presumed when plain films reveal (1) a foreign body, (2) associated soft-tissue swelling, (3) abnormal gas accumulation in the soft tissue of the neck, (4) and/or a loss of cervical lordosis. A CT scan of the neck, utilising extra fine cuts of $1 \mathrm{~mm}$ is the investigation of choice and is invaluable in confirming the presence of the foreign body. It also serves as a "road map" for localizing the foreign body. CT scans however are not without their drawbacks. It is sometimes impossible to tell if the foreign body is partially or completely extra luminal. A CT scan taken first without, and then with oral contrast can sometimes be useful. If the foreign body can be seen distinctly separate from the contrast in the lumen, it can be presumed to be completely extra luminal [3].

A foreign body should be suspected to have migrated extraluminally when esophagoscopy fails to identify a foreign body and post operative X-rays confirm it is still in the neck. Certain endoscopic findings-ulceration, edema, and laceration should arouse suspicion of migration. Having confirmed that the foreign body is extra luminal, exploration and removal of the foreign body via an external approach is recommended, to avoid life threatening complications. With the patient under anaesthesia, prior to commencing the exploration, a rigid laryngoscopy/ esophagoscopy should be repeated by an experienced surgeon. If a small portion of the foreign body has remained intraluminal, it may be identified and removed without the need for an open operation as happened in our case. If esophagoscopy proves unsuccessful, the exploration proceeds.

\section{References}

1. Jemerin EF, Amoff JS (1949) Foreign body in thyroid following perforation of esophagus surgery 25:52-59

2. Muhanna AA, et al (1990) Thyroid lobectomy for removal of a fish bone. J Laryngol Otol 104:511-512

3. Sethi DS, Stanley R (1992) Migrating foreign bodies in the upper digestive tract. Annals Academy of Medicine Singapore 21:3

4. Connolly AA, Birchall M, Walsh-Waring GP, Moore-Gillon V (1992) Ingested foreign bodies: Patient-guided localization is a useful clinical tool.Clin Otolaryngol Allied Sci 17: $520-524$

5. Shu MT, Leu YS (2001) Microscopic removal of an embedded foreign body from the hypopharynx :report of two cases. Ear Nose Throat J 80:889-890

6. Chee LW, Sethi DS (1999) Diagnostic and therapeutic approach to migrating foreign bodies. Ann Otol Rhinol Laryngol 108:177-180

7. Remsen K, Lawson W, Billet HF, Som ML (1983) Unusual presentations of penetrating foreign bodies of the upper aerodigestive tract. Ann Otol Rhinol Laryngol Suppl 105: $32-44$ 